Revue internationale P.M.E.

Économie et gestion de la petite et moyenne entreprise

\title{
Petites et moyennes entreprises (PME), facteur de renouveau du tissu industriel en Belgique?
}

\section{A.M. Kumps et R. Wtterwulghe}

Volume 1, numéro 1, 1988

URI : https://id.erudit.org/iderudit/1007876ar

DOI : https://doi.org/10.7202/1007876ar

Aller au sommaire du numéro

Éditeur(s)

Presses de l’Université du Québec

ISSN

0776-5436 (imprimé)

1918-9699 (numérique)

Découvrir la revue

Citer cet article

Kumps, A. \& Wtterwulghe, R. (1988). Petites et moyennes entreprises (PME), facteur de renouveau du tissu industriel en Belgique? Revue internationale P.M.E., 1(1), 97-114. https://doi.org/10.7202/1007876ar

\section{Résumé de l'article}

Cet article analyse le rôle joué par les PME en Belgique dans le renouvellement du tissu industriel et la création d'emplois. Les théories économiques développées en cette matière font l'objet d'une analyse empirique basée sur les données belges recensées durant la période 1977-1986. L'étude distingue les firmes selon leur appartenance sectorielle et leur classe d'emploi. Les performances des petites entreprises sont ainsi systématiquement comparées à celles des entités de moyenne et grande dimension. Il apparaît que les PME de par leur souplesse d'adaptation ont mieux résisté dans une économie en mutation caractérisée par une évolution technologique rapide créant de nouveaux interstices pour ce type d'entreprise. On retiendra qu'en Belgique, l'augmentation du nombre d'entreprises est essentiellement imputable à un taux de natalité positif dans la population des petites entreprises du secteur tertiaire qui enregistre, par ailleurs, une création nette d'emplois durant la période étudiée. 


\title{
Petites et moyennes entreprises (PME), facteur de renouveau du tissu industriel en Belgique?
}

\author{
A.M. KUMPS \\ Doyen de la Faculté des Sciences Economiques, Sociales et Politiques \\ Université catholique de Louvain \\ R. WTTERWULGHE, \\ Président de l'Institut d'Etudes Européennes \\ Université Catholique de Louvain
}

\begin{abstract}
RESUME
Cet article analyse le rôle joué par les PME en Belgique dans le renouvellement du tissu industriel et la création d'emplois. Les théories économiques développées en cette matière font l'objet d'une analyse empirique basée sur les données belges recensées durant la période 1977-1986. L'étude distingue les firmes selon leur appartenance sectorielle et leur classe d'emploi. Les performances des petites entreprises sont ainsi systématiquement comparées à celles des entités de moyenne et grande dimension. II apparaît que les PME de par leur souplesse d'adaptation ont mieux résisté dans une économie en mutation caractérisée par une évolution technologique rapide créant de nouveaux interstices pour ce type d'entreprise. On retiendra qu'en Belgique, l'augmentation du nombre d'entreprises est essentiellement imputable à un taux de natalité positif dans la population des petites entreprises du secteur tertiaire qui enregistre, par ailleurs, une création nette d'emplois durant la période étudiée.
\end{abstract}

\begin{abstract}
This article analyses the role played by small companies in the field of the renewal of industrial structure and the creation of jobs. An empiric study, based on the Belgian figures recorded from 1977 still 1986, deals with the economic theories developed in that context. The study divides the prims according to the sectors to which they belong and the kind of jobs. And so are the results of small scale companies systematically, compared with those of middle and large scale firms. It appears that firms of small and middle scale resisted better, thanks to their adaptation flexibility, in a changing economy characterized by a quick technological development. That development creates new openings for that kind of company. In summary, the increase of the number of companies is essentially due to a positive birthrate in the small scale firms of the tertiary sector, in Belgium - further - more, this sector registers a creation of jobs during the period of time considered.
\end{abstract}

\section{RESUMEN}

Este artículo analiza el papel desempeñado por las PME en Bélgica en lo que atañe a la renovación de la estructura industrial y a la creación de empleos. En este tema, las teorias económicas desarrolladas son objeto de una análisis empírica fundada en las cifras belgas registradas desde el año 1977 haste el año 1986. La investigación diferencia las empresas según su pertenencia sectorial y su tipo de empleo. Asi, los resultados de las pequeñas empresas están, cada vez, comparados con los de las medias y grandes empresa. Resulta que las PME, gracias a su flexibilidad de adaptación, han resistido mejor en una economía en evolución, caracterisada por un rapido desarrollo tecnológico que crea nuevas posibilidades para este tipo de empresa. Entonces, se destaca del estudio que, en Bélgica, el aumento del número de empresas está debido, en gran parte, a un indice de natalidad positivo en la populación de pequeñas empresas del sector terciaro. Ademàs, el sector terciario registra una clara creación de empleos desde el año 1977 hasta el año 1986. 
Petites et moyennes entreprises (PME),

La croissance économique rapide des Golden Sixties avait consacré le rôle de la grande entreprise. La crise et la troisième révolution industrielle ont progressivement mis en avant de la scène la place et le rôle socio-économique de la petite et moyenne entreprise dans les structures industrielles.

Cette évolution s'est traduite par un regain d'intérêt tant des scientifiques que des pouvoirs publics à l'égard des P.M.E. Dans les différents pays industrialisés, les études sur le rôle de la P.M.E. se sont multipliées. Néanmoins, l'analyse globale du phénomène de la petite dimension demeure encore fort peu développée. Trop souvent, les recherches privilégient un aspect particulier de la P.M.E. sans analyser les fonctions économiques que la petite dimension joue aujourd'hui. Or, à la lumière du développementéconomique actuel, laP.M.E. constitue, grâce à ses caractéristiques spécifiques, un facteur essentiel de transition d'une économie en mutation. Ce phénomène de croissance du nombre de P.M.E. auquel on assiste a parfois été présenté comme «une véritable rupture dans l'évolution naturelle du capitalisme» (Julien et Morel, 1986).

Cette transformation actuelle de l'économie résulte du passage de la récession à la reprise et surtout de la troisième révolution industrielle engendrée par le développement des technologies nouvelles.

\section{La P.M.E., un phénomène d'économie en mutation}

Les périodes de stabilité économique accompagnées d'une croissance sans bouleversement majeur des technologies ont favorisé le rôle de la grande dimension. «Big was still seen as beautiful and growth was seen as the key to the treasures of increased economies of scale» (Curran et Stanworth, 1984). L'obtention de rendements d'échelles et d'effets de synergie justifiait les concentrations et la grande taille. On s'interrogeait peu sur l'opportunité du maintien de la petite et moyenne dimension, dont l'importance semblait d'ailleurs régresser dans divers secteurs industriels. La disparition des P.M.E paraissait tellement préoccupante qu'en Angleterre la Commission BOLTON fut chargée d'en analyser les causes (Bolton, 1978). Dans ce pays, le nombre de petites entreprises du secteur manufacturier est passé de 66.000 unités en 1958 à 60.000 en 1963 alors que le nombre d'entreprises de grande dimension demeurait relativement constant -4.000 entités -.

Ce phénomène s'est vérifié en Belgique. Durant la période de croissance économique de 1960 à 1969, l'importance relative des firmes occupant moins de 50 personnes régresse légèrement, passant de $96,2 \%$ à $95,5 \%$ du total des firmes. Cette décroissance est essentiellement imputable aux entités employant moins de 5 personnes ${ }^{1}$.

1 O.N.S.S. rapports annuels et calculs propres 
Durant la période 1954-1963, on a par ailleurs observé une diminution relative de l'importance des petits établissements en termes d'emploi dans différents pays. En Grande-Bretagne, leur poids relatif passe de $33 \%$ à $31 \%$, en Allemagne, de $40 \%$ à $34 \%$, en France de 58 à $51 \%$ et en Norvège de 70 à $65 \%$ (Bolton, 1978).

Ces données confirment la décroissance de l'importance relative desP.M.E. dans des périodes de croissance stable.

Il n'est dès lors pas étonnant de constater que jusqu'à la fin des années soixante, la place de la petite et moyenne entreprise dans les structures industrielles fût relativement ignorée des économistes. Pour ces derniers, les forces du marché décident en conditionnant les firmes à travailler à leur taille optimale. Il est clair que dans cet esprit, les rendements d'échelles allaient conduire à un phénomène de concentration. C'est en s'appuyant sur ce même fondement que Marx prédisait l'arrivée du socialisme à travers la concentration capitaliste qui devait conduire à la lutte desclasses età la révolution(Marx, 1978). On sait que les pays qui suivirent cette voie aboutirent à un capitalisme d'Etat où la grande dimension à structure rigide est à la base de toute évolution économique.

L'ouvrage qui marqua certes le plus l'attitude des économistes et des décideurs politiques à l'égard de la grande dimension est celui de Shumpeter (1947) qui la prôna résolument comme un processus inéluctable et nécessaire : «It is not sufficient to argue that because perfect competition is impossible under modern conditions - or because it always has been impossible - the large-scale establishment or unit of control must be accepted as a necessary evil inseparable from the economic progress which it is prevented from sabotaging by the forces inherent in its productive apparatus. What we have got to accept is that it has come to be the most powerful engine in that progress and in particular of the long-run expansion of total output, not only in spite of, but to a considerable extent through, this strategy which looks so restrictive when viewed in the individual case and from the individual point of time».

On affirme aussi que la grande entreprise a une meilleure capacité de s'adapter aux changements de goûts des consommateurs, de prévoir et d'organiser la production et de résister aux crises économiques. Cette idée se trouve confirmée en 1957 par Galbraith dans son «American Capitalism». Elle est reprise dans ses ouvrages ultérieurs. Il affirme notamment, en 1967 : «The size of General Motors is in the service not of monopoly or the economics of scale but of planning. And for this planning-control of supply, control of demand provision of capital, minimisation of risk there is no clear upper-limit to the desirable size. It could be the bigger the better».

Dans cette optique qui domine les Golden Sixties, la grande dimension paraît non seulement inévitable mais aussi souhaitable. Les économies d'échelles obtenues dans divers secteurs conduisent à une diminution des coûts et à une réduction des prix. De la production à la chaîne des véhicules automobiles au super pétrolier géant, en passant par les grands circuits intégrés de la distribution, tout conduisait à accréditer l'hypothèse selon laquelle la petite dimension est vouée à un rôle d'épiphénomène dans l'économie moderne. 
La fin de la croissance des Golden Sixties, la phase de récession, puis l'amorce d'une reprise, et surtout la troisième révolution industrielle, remettent en cause ce postulat de l'inéluctable grande dimension.

On assiste à un véritable renversement de tendance. Le poids des P.M.E., qui s'allégeait d'année en année durant cette période, s'accroît progressivement avec la crise. Ce phénomène paraît général dans leséconomies des pays industrialisés. Ainsi, entre 1974 et 1980, l'industrie française a perdu 500.000 emplois; le recul a été beaucoup plus net pour les grandes entreprises, qui en ont perdu 350.000, soit $12 \%$ de leurs effectifs, que pour les entreprises de moins de 500 salariés où la perte est de 150.000, soit $4 \%$ (Delattre et Eymard Duvernay, 1984).

Quelques études avaient déjà insisté sur les faiblesses du gigantisme et contribué ainsi à amorcer la démystification de la grande entreprise. Elles mettaient en avant tantôt la plus grande rentabilité des actifs de la petite dimension (Bolton, 1978), tantôt le fait que les économies d'échelles techniques liées à la grande dimension s'accompagnaient de déséconomies d'échelles liées à un manque de flexibilité (Samuels et Smyth, 1968). D'autres, comme Bain en 1966 ou Scherer, ont montré que leséconomiesd'échelles techniques peuventêtre atteintes assez rapidement et que le seuil minimal d'efficience est obtenu pour des dimensions plus faibles. Des auteurs commeE. Penrose ont également mis en avant l'existence de diverses formes d'économies d'échelles accessibles à de petites et moyennes dimensions (Penrose, 1959).

Dans un monde où les progrès scientifiques et technologiques sont rapides, les occasions de faire du neuf ne manquent pas. Les grandes entreprises en retiennent certaines mais pas toutes et les opportunités induites d'une évolution technologique rapide créent des interstices nouveaux pour les P.M.E.

La troisième révolution industrielle modifie fondamentalement les conditions de la production et repose sur une souplesse de créativité et d'adaptation dont un rouage essentiel est la petite dimension.Celle-ci semble particulièrement apte à diffuser les progrès technologiques et à contribuer ainsi à la création d'emplois. La P.M.E. est aussi appelée à jouer un rôle dans le renouveau du tissu industriel.

Plus fondamentalement, on peut considérer que «la renaissance des petites et moyennes entreprises appartient aussi à la dynamique culturelle d'une transition d'une société en mutation» (Julien et Morel, 1986).

\section{La P.M.E., facteur de renouvellement du tissu industriel et source de création d'emplois ?}

Ces dernières années, plusieurs études se sont efforcées d'analyser le rôle et l'impact des P.M.E. dans les pays industrialisés à travers divers paramètres. Il n'est guère contestable que l'approche statistique se heurte en ce domaine à des difficultés dont la moindre n'est pas de définir et de cerner la P.M.E. à travers des données 
quantitatives. Certaines études sont sans doute à considérer avec réserve car elles lient systématiquement le phénomène de la P.M.E. à la notion d'établissement, en négligeant le concept d'entreprise qui seul devrait entrer en ligne de compte ${ }^{2}$.

Dans les pages qui suivent, nous nous interrogeons sur le rôle qu'ont joué les P.M.E. en Belgique durant ces dix dernières années dans le renouvellement du tissu industriel d'une part, et dans la création d'emplois d'autre part. Notre analyse statistique est basée sur le concept d'entreprise (Dupriez, Kumps et Wtterwulghe, 1987). Sont considérées comme «petites», les entreprises dont l'emploi est inférieur à 50, comme «moyennes», celles dont les effectifs sont compris entre 50 et 99 et «grandes», celles dont le nombre de salariés dépasse la centaine.

\subsection{P.M.E. et renouveau industriel}

Si durant les Golden Sixties, les petites entreprises semblaient parfois promises à une lente disparition, après plusieurs années de crise, elles paraissent au contraire dotées d'une meilleure résistance que les grandes.

En Belgique, les petites entreprises comptent aujourd'hui pour plus de $97 \%$ du total des entreprises. On observe par ailleurs, durant la période 1977-1986, un accroissement de l'importance relative de la petite dimension qui repose, comme le montre le tableau 1, sur une augmentation du nombre net de petites entreprises.

Tab. 1 Evolution des entreprises en Belgique suivant les classes d'emplol - $\Delta$ 1977-1986

\begin{tabular}{|c|c|c|c|c|}
\cline { 2 - 5 } \multicolumn{1}{c|}{} & 1 à 49 & 50 à 99 & $>100$ & TOTAL \\
\hline 1977 & 130.425 & 2.614 & 2.511 & 135.550 \\
\hline$\%$ & 96,2 & 1,9 & 1,9 & 100 \\
\hline 1986 & 139.634 & 1.796 & 2.009 & 143.439 \\
\hline$\%$ & 97,4 & 1,2 & 1,4 & 100 \\
\hline$\Delta 77-86$ & $\begin{array}{r}+9.209 \\
+7,1 \%\end{array}$ & $\begin{array}{c}-818 \\
-31,3 \%\end{array}$ & $\begin{array}{r}-502 \\
-20,0 \%\end{array}$ & $\begin{array}{c}+7.889 \\
+5,8 \%\end{array}$ \\
\hline
\end{tabular}

Le tableau indique une création nette de 9.209 unités au sein de la population des petites entreprises correspondant à un taux de croissance de plus de $7 \%$. Les autres entreprises se caractérisent par une dénatalité nette très sensible. La population

2 O.N.S.S., Rapports annuels 
des moyennes et grandes entreprises diminue respectivement de 818 et 502 unités, ce qui représente une décroissance relative de $31,3 \%$ pour les premières et de $20,0 \%$ pour les secondes.

L'analyse de l'évolution annuelle du mouvement démographique des entreprises révèle une diminution continue du nombre des moyennes et grandes entreprises durant la période. Les firmes employant moins de 50 personnes connaissent une évolution un peu plus différentiée, comme l'illustre le tableau 2.

Tab. 2 Evolution annuelle des entreprises

\begin{tabular}{|c|c|c|c|c|}
\cline { 2 - 5 } \multicolumn{1}{c|}{} & 1 à 49 & 50 à 99 & $>100$ & Total \\
\hline $1977-78$ & $+1,70$ & $-3,1$ & $-1,8$ & $+1,5$ \\
$1978-79$ & $+0,2$ & $-5,4$ & $-3,0$ & $+0,1$ \\
$1979-80$ & $+0,3$ & $-0,3$ & $-2,9$ & $+0,3$ \\
$1980-81$ & - & $-3,2$ & - & - \\
$1981-82$ & $-0,2$ & $-6,0$ & $-3,8$ & $-0,4$ \\
$1982-83$ & $+0,70$ & $-5,5$ & $-4,5$ & $+0,5$ \\
$1983-84$ & $+1,50$ & $-5,5$ & $-3,3$ & $+1,3$ \\
$1984-85$ & $+1,3$ & $-3,5$ & $-1,7$ & $+1,2$ \\
$1985-86$ & $+1,4$ & $-4,1$ & $-0,9$ & $+1,2$ \\
\hline$\Delta 1977-86$ & $+7,1$ & $-31,3$ & $-20,0$ & $+5,8$ \\
\hline
\end{tabular}

A la lecture du tableau, on constate que la croissance de la population des petites entreprises n'est pas continue. Après une augmentation importante en 1978, le nombre d'unités de petite dimension a tendance à se tasser jusqu'en 1980. En 1982, la classe de 1 à 49 travailleurs enregistre un recul dû vraisemblablementà la mauvaise conjoncture de l'année 1981 qui s'était caractérisée par une diminution du Produit National Brut en termes réels de $1,4 \%{ }^{3}$. La reprise se manifeste dès 1983 et le mouvement de créations nettes de petites entités se poursuit jusqu'en 1986 à un rythme avoisinant $1,5 \%$ par an.

Grâce à leur souplesse et leur faculté d'adaptation rapide, les petites entreprises sont donc les seules à avoir saisi, durant ces dix années, les opportunités offertes par un climat économique plus favorable.

Sur la période analysée, il est important de relever que la croissance nette du nombre d'entreprises de petite dimension a pu compenser la dénatalité enregistrée auprès des autres entreprises. Cela semble confirmer que les performances des petites

3 P.N.B. à prix constant de 1980. 
entreprises témoignent non seulement d'une meilleure résistance à la crise mais aussi d'une dynamique de renouvellement du tissu industriel.

Après avoir mis en lumière l'importance et l'évolution favorable de la petite dimension en Belgique, nous affinerons notre analyse en prenant en considération l'appartenance sectorielle des firmes. Comme l'indique le tableau 3, l'importance relative de la petite dimension s'accroît depuis 1977, quel que soit le secteur considéré.

Tab. 3 Part relative des entreprises

selon leur appartenance sectorielle $-\Delta$ 1977-1986

\begin{tabular}{|c|c|c|c|c|c|}
\hline & & 1 à 49 & 50 à 99 & $>100$ & TOTAL \\
\hline \multirow{2}{*}{ Primaire } & 77 & 99,8 & 0,2 & - & 100 \\
\hline & 86 & 99,9 & 0,1 & - & 100 \\
\hline$\Delta 77-86$ & & $-0,2$ & $-50,0$ & - & $-0,3$ \\
\hline \multirow{2}{*}{ Secondaire } & 77 & 93,1 & 3,3 & 3,6 & 100 \\
\hline & 86 & 94,7 & 2,4 & 2,9 & 100 \\
\hline$\Delta 77-86$ & & $-8,8$ & $-36,1$ & $-26,5$ & $-10,3$ \\
\hline \multirow[t]{2}{*}{ Tertiaire } & 77 & 98,0 & 1,1 & 0,9 & 100 \\
\hline & 86 & 98,5 & 0,8 & 0,7 & 100 \\
\hline$\Delta 77-86$ & & $+16,8$ & $-22,3$ & $-4,0$ & $+16,2$ \\
\hline \multirow[t]{2}{*}{ TOTAL } & 77 & 96,2 & 1,9 & 1,9 & 100 \\
\hline & 86 & 97,4 & 1,2 & 1,4 & 100 \\
\hline$\Delta 77-86$ & & $+7,1$ & $-31,3$ & $-20,0$ & $+5,8$ \\
\hline
\end{tabular}

On constate que tout en demeurant moins bien représentées que dans le primaire et le tertiaire, la part relative des petites entreprises s'accroît de manière significative dans le secteur secondaire. Les performances particulièrement mauvaises 
des moyennes et grandes entreprises expliquent cette évolution. Par ailleurs, la forte croissance du nombre de petites entités dans le tertiaire ne contribue qu'à un faible renforcement de leur importance relative, les autres classes de dimension offrant une meilleure résistance dans ce secteur.

Le tableau 4 illustre la répartition sectorielle des entreprises en Belgique par classe d'emploi en 1986 et indique les évolutions relatives observées sur la période 1977-1986.

Le tableau met en lumière l'importance du secteur tertiaire. En 1986, 65,6 \% de la population totale des entreprises belges sont situés dans ce secteur contre 31,3\% dans le secondaire, alors que 9 ans plus tôt, ces pourcentages étaient respectivement de $59,4 \%$ et $37 \%$. Parallèlement, on observe une diminution de $10,3 \%$ du nombre d'entreprises du secondaire et une augmentation de $16,2 \%$ de la population des firmes du tertiaire. On constate donc que la Belgique suit le mouvement observé dans d'autres économies occidentales qui aboutit au renforcement progressif du secteur tertiaire au détriment du secteur secondaire mais aussi et surtout que le mouvement repose exclusivement sur la petite dimension. Elle joue un rôle fondamental dans le processus de renouvellement.

La croissance de plus de $16 \%$ du nombre d'entreprises du secteur tertiaire s'explique uniquement par la natalité des petites entreprises dont le nombre s'accroît de $16,9 \%$ alors que les moyennes et les grandes entreprises diminuentrespectivement de $-22,3 \%$ et $-4,4 \%$ sur la période $1977-1986$. L'augmentation du nombre d'entreprises de petite dimension dans le secteur tertiaire est à mettre principalement à l'actif des petites unités du secteur «Commerce, restauration et hébergement, réparations» qui enregistre un taux de renouvellement de $20,4 \%$ et de celles du secteur des «Institutions de crédit, assurances, services fournis aux entreprises et location» dont la hausse s'élève à 32,6\%.

Les taux de croissance annuels des petites entreprises du secteur tertiaire s'avèrent positifs pour chacune des années étudiées.

Au niveau du secteur secondaire, la population des entreprises de tous les sous-secteurs est en décroissance et ce, quelle que soit la dimension des entreprises. On observe cependant que l'ampleur relative de la dénatalité est fonction croissante de la dimension des firmes. Elle est de - 8,8\% pour les petites entreprises, de - 36,1\% pour les moyennes et de $-26,5 \%$ pour les firmes occupant plus de 100 travailleurs.

L'analyse de l'évolution annuelle du nombre d'entreprises révèle une diminution continue du nombre de petites entités dans le secteur secondaire jusqu'en 1984. Depuis cette année, le taux de natalité redevient positif. Le climat économique plus optimiste s'est donc traduit par une reprise de la volonté d' «entreprendre» dans le secteur industriel. 
Tab.4 Répartition des entreprises en 1986 et évolution par taille et grands secteurs d'activité $-\Delta$ 1977-1986

\begin{tabular}{|c|c|c|c|c|c|c|c|c|}
\hline \multirow[b]{3}{*}{ Agriculture } & \multirow{2}{*}{\multicolumn{2}{|c|}{$\frac{1 \text { à } 49}{\% \Delta 77-86}$}} & \multirow{2}{*}{\multicolumn{2}{|c|}{$\frac{50 \text { à } 99}{\% \Delta 77-86}$}} & \multirow{2}{*}{\multicolumn{2}{|c|}{$\frac{>100}{\% \Delta 77-86}$}} & \multicolumn{2}{|c|}{ TOTAL } \\
\hline & & & & & & & $\%$ & $\Delta 77-86$ \\
\hline & 3,2 & $-0,2$ & 0,3 & $-50,0$ & - & - & 3,1 & $-0,3$ \\
\hline PRIMAIRE & 3,2 & $-0,2$ & 0,3 & $-50,0$ & - & - & 3,1 & $-0,3$ \\
\hline $\begin{array}{l}\text { Energie et } \\
\text { eau }\end{array}$ & 0,0 & $-34,6$ & 0,1 & $-66,6$ & 0,9 & $-35,1$ & 0,0 & $-37,1$ \\
\hline $\begin{array}{l}\text { Extraction et } \\
\text { Indust. chim. }\end{array}$ & 1,2 & $-22,3$ & 5,9 & $-30,3$ & 10,6 & $-18,3$ & 1,4 & $-22,4$ \\
\hline $\begin{array}{l}\text { Métallurgie et } \\
\text { mécanique }\end{array}$ & 3,5 & $-2,0$ & 12,8 & $-24,1$ & 16,4 & $-20,7$ & 3,8 & $-4,6$ \\
\hline $\begin{array}{l}\text { Autres indust. } \\
\text { manufactur. }\end{array}$ & 10,6 & $-8,9$ & 28,9 & $-34,0$ & 27,6 & $-27,4$ & 11,1 & $-10,8$ \\
\hline Construction & 15,2 & $-8,7$ & 12,4 & $-49,2$ & 9,7 & $-36,2$ & 15,0 & $-9,8$ \\
\hline SECONDAIRE & 30,5 & $-8,8$ & 60,1 & $-36,1$ & 65,2 & $-26,5$ & 31,3 & $-10,3$ \\
\hline Commerce & 48,4 & $+20,4$ & 20,8 & $-27,9$ & 15,1 & $-10,3$ & 47,6 & $+19,8$ \\
\hline Transports & 4,1 & $+9,5$ & 6,3 & $+2,7$ & $-4,4$ & $+2,3$ & 4,1 & $+9,2$ \\
\hline $\begin{array}{l}\text { Instit. crédit } \\
\text { Assurance, } \\
\text { location }\end{array}$ & 6,9 & $+32,6$ & 7,3 & $-10,9$ & 9,3 & $+10,6$ & 7,0 & $+31,3$ \\
\hline $\begin{array}{l}\text { Serv. publics } \\
\text { autres }\end{array}$ & 6,9 & $-9,5$ & 5,2 & $-33,1$ & 5,9 & $-10,5$ & 6,9 & $-9,8$ \\
\hline TERTIAIRE & 66,3 & $+16,9$ & 39,6 & $-22,3$ & 34,8 & $-4,4$ & 65,6 & $+16,2$ \\
\hline TOTAL & $\begin{array}{l}100 \% \\
(139.6\end{array}$ & $\begin{array}{l}+7,1 \\
34)\end{array}$ & $\begin{array}{l}100 \% \\
(1.796\end{array}$ & $-31,3$ & $\begin{array}{l}100 \% \\
(2.009)\end{array}$ & $-20,0$ & $\begin{array}{l}100 \% \\
(143.4\end{array}$ & $\begin{array}{l}+5,8 \\
439)\end{array}$ \\
\hline
\end{tabular}




\section{$2.2 \quad$ P.M.E. et création d'emplois}

Un indicateur important de la vitalité des firmes est celui de l'emploi. En période de récession et de chômage, ce paramètre a, à plusieurs reprises, été mis en exergue. Diverses études se sont attachées à déterminer si les P.M.E. étaient créatrices d'emplois (Greffe, 1984, Delattre, 1986, Fothergill et Gudgin, 1979, Storey, 1980).

Le point de départ de l'analyse de la contribution des P.M.E. à la création d'emplois demeure l'étude réalisée par P. Birch du M.I.T. Selon celle-ci, $66 \%$ des créations nettes d'emplois aux Etats-Unis sur la période 1969-1976 sont le fait d'entreprises de moins de 20 salariés appartenant principalement au secteur des services. Les entreprises de moins de 100 salariés interviennent par ailleurs, selon $P$. Birch, pour $82 \%$ des créations d'emploi bien qu'elles ne représentent que $40 \%$ des effectifs employés. Cette analyse de P. Birch n'est pas sans soulever des critiques quant aux données et à la méthodologie utilisées. Elle a néanmoins eu le mérite de mettre en lumière une tendance jusque là trop souvent négligée, à savoir le rôle de la P.M.E. en matière de création d'emplois. Cette étude a créé une émulation qui a conduit à analyser le problème dans d'autres pays.

En Belgique, les statistiques relatives à l'évolution de l'emploi par classe de dimension des firmes et par secteur d'activité ne sont disponibles qu'au niveau du secteur privé. L'emploi public, en ce compris l'enseignement, ne peut en effet être ventilé en fonction de l'importance des entreprises en termes d'emploi. Comme le secteur public se caractérise souvent par la grande dimension des entités qui le composent, les tendances observées dans le secteur privé paraissent significatives pour une analyse de la contribution des P.M.E. à la création d'emplois. Nous avons en conséquence étudié l'évolution de l'emploi procuré par les entreprises privées de diverses dimensions durant la période 1978-1986, seules années pour lesquelles existe une ventilation sectorielle.

Les petites entreprises occupent, en 1986, $39 \%$ de l'emploi privé, et comme l'indique le tableau 5, leur importance relative en tant que foumisseurs d'emploi augmente durant la période.

Le tableau met en lumière les mauvaises performances de l'emploi privé dont témoigne, par ailleurs, la montée préoccupante du chômage en Belgique au cours de ces dernières années. On constate néanmoins que les pertes d'emplois sont beaucoup moins importantes dans la population des petites entreprises. Elles y atteignent $1,6 \%$ contre respectivement $19,3 \%$ et $11,2 \%$ dans la moyenne et grande dimension. Nous ajouterons qu'une ventilation plus fine des diverses catégories d'entreprises indique que les firmes occupant de 1 à 4 personnes enregistrent une augmentation de leurs effectifs employés de 6,4\% durant la période 78-86. L'analyse de l'évolution annuelle de l'emploi confirme, comme le montre le tableau 6, la meilleure résistance de la petite dimension. 
Tab. 5 Evolution de l'emploi privé en Belgique suivant les classes d'emplol - $\Delta$ 1978-1986 (`)

\begin{tabular}{|c|c|c|c|c|}
\cline { 2 - 5 } \multicolumn{1}{c|}{} & 1 à 49 & 50 à 99 & $>100$ & TOTAL \\
\hline 1978 & 773.257 & 191.106 & 1.166 .552 & 2.130 .915 \\
\hline$\%$ & 36,3 & 9,0 & 54,7 & 100 \\
\hline 1986 & 761.060 & 154.239 & 1.035 .516 & 1.950 .815 \\
\hline$\%$ & 39,0 & 7,9 & 53,1 & 100 \\
\hline$\Delta 78-86$ & $\begin{array}{c}-12.217 \\
-1,6 \%\end{array}$ & $\begin{array}{c}-36.867 \\
-19,3 \%\end{array}$ & $\begin{array}{c}-131.036 \\
-11,2 \%\end{array}$ & $\begin{array}{c}-180.120 \\
-8,5 \%\end{array}$ \\
\hline
\end{tabular}

Tab. 6 Evolution annuelle de l'emploi privé

\begin{tabular}{|c|c|c|c|c|c|}
\cline { 2 - 6 } \multicolumn{1}{c|}{} & 1 à 4 & 1 à 49 & 50 à 99 & $>100$ & TOTAL \\
\hline $1978-79$ & $+1,7$ & - & $-0,3$ & $-0,5$ & $-0,2$ \\
$1979-80$ & $+0,6$ & $-0,1$ & $-2,8$ & $-0,5$ & $-0,8$ \\
$1980-81$ & $+0,3$ & $-2,9$ & $-5,1$ & $-4,5$ & $-4,0$ \\
$1981-82$ & $+0,3$ & $-1,3$ & $-5,3$ & $-3,2$ & $-2,7$ \\
$1982-83$ & $+0,6$ & $-0,7$ & $-4,7$ & $-2,0$ & $-1,7$ \\
$1983-84$ & $+0,8$ & $-0,3$ & $-0,7$ & $-0,8$ & $-0,6$ \\
$1984-85$ & $+1,1$ & $+2,1$ & $-2,2$ & $+0,3$ & $+0,8$ \\
$1985-86$ & $+0,9$ & $+2,7$ & $-0,5$ & $-0,6$ & $+0,6$ \\
\hline \multirow{2}{*}{$\Delta 78-86$} & $+6,4$ & $-1,6$ & $-19,3$ & $-11,2$ & $-8,5$ \\
\hline
\end{tabular}

Il est frappant de constater que les toutes petites entités comptant moins de 5 personnes contribuent chaque année à la création d'emplois. D'une manière générale, les petites entreprises occupant moins de 50 travailleurs enregistrent des pertes d'emplois relativement moindres que celles observées dans la moyenne et grande dimension. Elles semblent donc subir de manière moins accusée les effets de la crise du début des années 80 . Depuis 1985, elles se caractérisent par un taux de

4 Source : O.N.S.S., Statistiques "employeurs" non régionalisées et calculs propres. 
renouvellement positif de l'emploi et paraissent ainsi être les premières à s'être inscrites avec détermination dans un climat économique où se manifestent les signes avant-coureurs d'une reprise des activités.

L'analyse du rôle joué par les petites entreprises dans le renouveau du tissu industriel en Belgique a mis en avant des évolutions sectorielles différentiées. Nous nous proposons de vérifier si elles existent également en termes de créations d'emplois.

L'importance relative de l'emploi fourni par les petites entreprises privées augmente dans les trois grands secteurs de l'économie, comme l'atteste le tableau 7.

Tab. 7 Part relative de l'emploi procuré par les entreprises privées par grands secteurs d'activité - $\Delta 78-86$

\begin{tabular}{|c|c|c|c|c|c|}
\hline & & 1 à 49 & 50 à 99 & $>100$ & TOTAL \\
\hline \multirow[t]{2}{*}{ Primaire } & 78 & 97,7 & 3,3 & - & 100 \\
\hline & 86 & 97,4 & 2,6 & - & 100 \\
\hline$\Delta 78-86$ & & $+4,4$ & $-13,6$ & - & $+3,8$ \\
\hline \multirow[t]{2}{*}{ Secondaire } & 78 & 27,6 & 9,0 & 63,4 & 100 \\
\hline & 86 & 29,4 & 8,4 & 62,2 & 100 \\
\hline$\Delta 78-86$ & & $-17,5$ & $-27,6$ & -24 & $-22,5$ \\
\hline \multirow[t]{2}{*}{ Tertiaire } & 78 & 47,1 & 9,0 & 43,9 & 100 \\
\hline & 86 & 47,3 & 7,5 & 45,2 & 100 \\
\hline$\Delta 78-86$ & & $+10,7$ & $-8,1$ & $+13,3$ & $+10,2$ \\
\hline \multirow[t]{2}{*}{ TOTAL } & 78 & 36,3 & 9,0 & 54,7 & 100 \\
\hline & 86 & 39,0 & 7,9 & 53,1 & 100 \\
\hline$\Delta 78-86$ & & $-1,6$ & $-19,3$ & $-11,2$ & $-8,5$ \\
\hline
\end{tabular}


On constate que l'importance relative de l'emploi fourni par les petites entreprises du secteur secondaire s'accroît durant la période de près de $2 \%$. Cette évolution est due au fait que les pertes d'emplois des moyennes et grandes entreprises se sont avérées plus importantes que celles observées dans la petite dimension. On retrouve ici la même explication que celle fournie au niveau du mouvement démographique des entreprises. Dans un mouvement de déclin, qu'il s'agisse du nombre d'entreprises ou de l'emploi, la petite dimension résiste mieux dans le secteur secondaire que la moyenne et la grande. A ce stade, il convient de préciser que l'évolution du nombre d'entreprises privées est fort similaire à celle de la population totale des entreprises ${ }^{5}$.

Auniveau du secteur tertiaire, la petite dimension fournit proportionnellement le plus d'emplois. Malgré une hausse de près de $11 \%$ de l'emploi procuré durant la période, 1 'importance relative des petites entreprises augmente néanmoins très peu... La croissance de l'emploi privé des grandes entreprises du secteur tertiaire dépasse les $13 \%$ et contribue à accroître leur part relative dans l'emploi de plus de $1 \%$. Avec un taux de natalité net de 3,9\% et un taux de croissance de l'emploi de $13,3 \%$, les grandes entreprises du secteur tertiaire privé s'inscrivent dans le mouvement de concentration déjà observé au niveau de la grande distribution et du secteur financier. Les petites entreprises résistent cependant bien dans les services, en affichant un taux de renouvellement net de $14 \%$ et une augmentation de l'emploi de l'ordre de $11 \%$.

Le tableau 8 illustre la répartition de l'emploi privé par taille d'entreprises et grands secteurs d'activité en 1986. Il indique également l'évolution observée sur la période 1978-1986.

$5 \quad$ Entreprises privées

\begin{tabular}{|c|c|c|c|c|c|}
\hline & & 1 à 49 & 50 à 99 & $>100$ & TOTAL \\
\hline \multirow[t]{2}{*}{ I } & $\begin{array}{l}78 \\
86\end{array}$ & $\begin{array}{l}99,9 \\
99,9\end{array}$ & $\begin{array}{l}0,1 \\
0,1\end{array}$ & - & $\begin{array}{l}100 \% \\
100 \%\end{array}$ \\
\hline & $\Delta 78-86$ & - & - & - & - \\
\hline \multirow[t]{2}{*}{ II } & $\begin{array}{l}78 \\
86\end{array}$ & $\begin{array}{l}93,5 \\
94,7\end{array}$ & $\begin{array}{l}3,1 \\
2,5\end{array}$ & $\begin{array}{l}3,4 \\
2,8\end{array}$ & $\begin{array}{l}100 \\
100\end{array}$ \\
\hline & $\Delta 78-86$ & $-10,8$ & $-29,7$ & $-25,0$ & $-11,9$ \\
\hline \multirow[t]{2}{*}{ III } & $\begin{array}{l}78 \\
86\end{array}$ & $\begin{array}{l}97,7 \\
98,1\end{array}$ & $\begin{array}{l}1,2 \\
0,9\end{array}$ & $\begin{array}{l}1,1 \\
1,0\end{array}$ & $\begin{array}{l}100 \\
100\end{array}$ \\
\hline & $\Delta 78-86$ & +14 & $-9,1$ & $+3,9$ & $+13,6$ \\
\hline TOTAL & $\begin{array}{l}78 \\
86\end{array}$ & $\begin{array}{l}96,4 \\
97,2\end{array}$ & $\begin{array}{l}1,8 \\
1,4\end{array}$ & $\begin{array}{l}1,8 \\
1,4\end{array}$ & $\begin{array}{l}100 \\
100\end{array}$ \\
\hline & $\Delta 78-86$ & $+5,9$ & $-20,8$ & $-13,5$ & +5 \\
\hline
\end{tabular}


Tab. 8 Répartition de l'emplol privé en 1986 et évolution par taille d'entreprise et grands secteurs d'activité $-\Delta$ 1978-1986

\begin{tabular}{|c|c|c|c|c|c|c|c|c|}
\hline \multirow[b]{3}{*}{ Agriculture } & \multirow{2}{*}{\multicolumn{2}{|c|}{$\frac{1 \text { à } 49}{\% \Delta 78-86}$}} & \multirow{2}{*}{\multicolumn{2}{|c|}{$\frac{50 \text { à } 99}{\% \Delta 78-86}$}} & \multirow{2}{*}{\multicolumn{2}{|c|}{$\frac{>100}{\% \Delta 78-86}$}} & \multicolumn{2}{|c|}{ TOTAL } \\
\hline & & & & & & & $\%$ & $\Delta 78-86$ \\
\hline & 1,6 & $+4,4$ & 0,2 & $-13,6$ & - & - & 0,6 & $+3,8$ \\
\hline PRIMAIRE & 1,6 & $+4,4$ & 0,2 & $-13,6$ & - & - & 0,6 & $+3,8$ \\
\hline $\begin{array}{l}\text { Energie et } \\
\text { eau }\end{array}$ & 0,04 & $+58,0$ & 0,1 & $-24,0$ & 4,1 & $-16,4$ & 2,2 & $-16,1$ \\
\hline $\begin{array}{l}\text { Extraction et } \\
\text { Indust. chim. }\end{array}$ & 2,1 & $-20,5$ & 5,2 & $-20,6$ & 14,2 & $-25,1$ & 8,8 & $-24,5$ \\
\hline $\begin{array}{l}\text { Métallurgie et } \\
\text { mécanique }\end{array}$ & 5,9 & $-0,9$ & 11,3 & $-10,6$ & 19,1 & $-19,6$ & 13,3 & $-16,3$ \\
\hline $\begin{array}{l}\text { Autres indust. } \\
\text { manufactur. }\end{array}$ & 14,8 & $-12,3$ & 24,7 & $-23,5$ & 15,3 & $-19,7$ & 15,9 & $-17,7$ \\
\hline Construction & 13,5 & $-27,2$ & 10,0 & $-48,2$ & 3,7 & $-50,6$ & 8,0 & $-37,0$ \\
\hline SECONDAIRE & 36,3 & $-17,5$ & 51,3 & $-27,6$ & 56,4 & $-24,0$ & 48,2 & $-22,5$ \\
\hline Commerce & 34,7 & $+6,4$ & 17,1 & $-25,2$ & 12,8 & $-1,3$ & 21,7 & $+1,3$ \\
\hline Transports & 5,8 & $+21,9$ & 5,5 & $+18,9$ & 3,4 & $-10,6$ & 4,5 & $+6,2$ \\
\hline $\begin{array}{l}\text { Instit. crédit } \\
\text { Assurance, } \\
\text { location }\end{array}$ & 9,4 & $+17,2$ & 8,6 & $+8,8$ & 13,3 & $+30,1$ & 11,4 & $+24,3$ \\
\hline Autres services & 12,2 & $+14,1$ & 17,4 & $-0,4$ & 14,1 & $+22,9$ & 13,6 & $+17,0$ \\
\hline TERTIAIRE & 62,1 & $+10,7$ & 48,5 & $-8,1$ & 43,6 & $+13,3$ & 51,2 & $+10,2$ \\
\hline TOTAL & $100 \%$ & $-1,6$ & $100 \%$ & $-19,3$ & $100 \%$ & $-11,2$ & $100 \%$ & $-8,5$ \\
\hline
\end{tabular}


A la lecture du tableau, on constate qu'en 1986, plus de $51 \%$ de l'emploi privé total est localisé dans le secteur des services. Ce taux avoisinerait les $66 \%$ si l'on avait pu intégrer l'emploi public. La prise en considération de la dimension des firmes indique, par ailleurs, que les petites entités occupent proportionnellement plus d'emplois dans le secteur tertiaire que les entreprises de moyenne et grande dimension. La part relative de l'emploi tertiaire s'établit en effet à $62,1 \%$ dans les firmes de moins de 50 travailleurs contre respectivement $48,5 \%$ et $43,6 \%$ dans la classe de 50 à 99 et celle de plus de 100 travailleurs. Parallèlement à l'accroissement relatif de l'importance de l'emploi tertiaire, on observe une diminution de l'emploi secondaire de $22,5 \%$.

La croissance de l'emploi tertiaire repose sur la création nette d'emplois par les firmes de petite dimension et ce, quel que soit leur sous-secteur d'appartenance... Elle s'explique aussi par la création importante de postes de travail dans les grandes entreprises du secteur financier et des services divers aux particuliers. La diminution de l'emploi secondaire s'avère, par ailleurs, relativement moins importante dans les firmes de petite dimension : - 17,5\% contre respectivement - 27,6 et - $24 \%$ dans les moyennes et grandes entreprises. On épinglera toutefois qu'en 1985 et 1986, les entités occupant moins de 50 personnes dans le secteur secondaire ont, contrairement aux firmes de plus grande dimension, enregistré des taux de renouvellement positifs de l'emploi.

Le tableau 9 compare les taux de variation annuels des employeurs privés et de l'emploi qu'ils procurent sur la période 1978-1986. Il distingue les firmes selon leur dimension et leur appartenance sectorielle. Le secteur primaire, non significatif, n'est pas repris.

Le tableau met en lumière le rôle joué depuis 1985 par les P.M.E. en Belgique dans la création d'emplois, qu'il s'agisse du secteur secondaire ou tertiaire.

A partir de l'année 1985 , le secteur industriel se caractérise par une augmentation du nombre de P.M.E. et de l'emploi qu'elles procurent. Le taux de création d'emplois s'avère, par ailleurs, supérieur à celui du renouvellement de la population des entités de petite dimension. Les P.M.E. industrielles, contrairement aux moyennes et grandes entreprises de ce secteur, contribuent en conséquence à créer de l'emploi.

Le secteur tertiaire se distingue durant toute la période analysée par une croissance du nombre de petites entités et de l'emploi qu'elles fournissent. Depuis 1985, on constate néanmoins que le taux de création d'emplois dépasse celui de la natalité des petites entreprises. Dans ce même secteur, on assiste durant les quatre dernières années à un mouvement de concentration au sein des grandes entreprises, le taux de croissance de l'emploi étant nettement supérieur à celui de la création de firmes.

La prise en considération de l'ensemble des secteurs indique que seules les petites entreprises se sont, au cours des années 1985 et 1986, distinguées par un taux de création d'emplois supérieur à celui observé au niveau du renouvellement de la population des firmes de petite dimension. 
Tab. 9 Taux de variation annuels des entreprises privées et de l'emploi procuré

\begin{tabular}{|c|c|c|c|c|c|c|c|c|c|}
\hline & 79 & 80 & 81 & 82 & 83 & 84 & 85 & 86 & $\Delta 78 / 86$ \\
\hline $\begin{array}{l}\text { Secondaire } \\
1 \text { à } 49 \\
\text { ENTR. } \\
\text { EMPLOI }\end{array}$ & $\begin{array}{l}-0,9 \\
-2,5\end{array}$ & $\begin{array}{l}-1,5 \\
-3,2\end{array}$ & $\begin{array}{l}-3,6 \\
-6,6\end{array}$ & $\begin{array}{l}-3,6 \\
-4,7\end{array}$ & $\begin{array}{l}-1,7 \\
-2,8\end{array}$ & $\begin{array}{l}-1,4 \\
-2,3\end{array}$ & $\begin{array}{l}0,5 \\
1,1\end{array}$ & $\begin{array}{l}1,0 \\
2,3\end{array}$ & $\begin{array}{l}-10,8 \\
-17,5\end{array}$ \\
\hline $\begin{array}{l}50 \text { à } 99 \\
\text { ENTR. } \\
\text { EMPLOI }\end{array}$ & $\begin{array}{l}0,6 \\
0,5\end{array}$ & $\begin{array}{l}-5,7 \\
-4,9\end{array}$ & $\begin{array}{l}-6,6 \\
-7,0\end{array}$ & $\begin{array}{l}-7,7 \\
-7,8\end{array}$ & $\begin{array}{l}-7,4 \\
-7,1\end{array}$ & $\begin{array}{l}-2,0 \\
-0,6\end{array}$ & $\begin{array}{l}-3,1 \\
-3,5\end{array}$ & $\begin{array}{l}-2,3 \\
-1,0\end{array}$ & $\begin{array}{l}-27,0 \\
-27,6\end{array}$ \\
\hline $\begin{array}{c}>100 \\
\text { ENTR. } \\
\text { EMPLOI }\end{array}$ & $\begin{array}{l}-4,2 \\
-2,2\end{array}$ & $\begin{array}{l}-1,4 \\
-1,7\end{array}$ & $\begin{array}{l}-5,9 \\
-6,5\end{array}$ & $\begin{array}{l}-4,2 \\
-4,8\end{array}$ & $\begin{array}{l}-4,5 \\
-3,6\end{array}$ & $\begin{array}{l}-4,0 \\
-2,7\end{array}$ & $\begin{array}{r}0,9 \\
-2,4\end{array}$ & $\begin{array}{l}-4,9 \\
-2,9\end{array}$ & $\begin{array}{l}-25,0 \\
-24,0\end{array}$ \\
\hline $\begin{array}{l}\text { TOTAL } \\
\text { ENTR. } \\
\text { EMPLOI }\end{array}$ & $\begin{array}{l}-1,2 \\
-2,1\end{array}$ & $\begin{array}{l}-1,6 \\
-2,4\end{array}$ & $\begin{array}{l}-3,8 \\
-6,6\end{array}$ & $\begin{array}{l}-3,7 \\
-5,1\end{array}$ & $\begin{array}{l}-2,0 \\
-3,7\end{array}$ & $\begin{array}{l}-1,5 \\
-2,4\end{array}$ & $\begin{array}{l}-0,5 \\
-1,5\end{array}$ & $\begin{array}{r}0,7 \\
-1,2\end{array}$ & $\begin{array}{l}-11,9 \\
-22,5\end{array}$ \\
\hline $\begin{array}{l}\text { Tertiaire } \\
1 \text { à } 49 \\
\text { ENTR. } \\
\text { EMPLOI }\end{array}$ & $\begin{array}{l}2,7 \\
2,0\end{array}$ & $\begin{array}{l}1,5 \\
0,8\end{array}$ & $\begin{array}{r}0,7 \\
-0,3\end{array}$ & $\begin{array}{l}0,9 \\
0,9\end{array}$ & $\begin{array}{l}1,7 \\
0,6\end{array}$ & $\begin{array}{l}1,9 \\
0,8\end{array}$ & $\begin{array}{l}1,9 \\
2,7\end{array}$ & $\begin{array}{l}1,9 \\
2,9\end{array}$ & $\begin{array}{l}+14,0 \\
+10,7\end{array}$ \\
\hline $\begin{array}{l}50 \text { à } 99 \\
\text { ENTR. } \\
\text { EMPLOI }\end{array}$ & $\begin{array}{r}-0,8 \\
0,1\end{array}$ & $\begin{array}{l}0,5 \\
0,1\end{array}$ & $\begin{array}{l}-3,5 \\
-2,5\end{array}$ & $\begin{array}{l}-1,2 \\
-2,3\end{array}$ & $\begin{array}{l}-2,2 \\
-2,0\end{array}$ & $\begin{array}{l}-1,7 \\
-0,8\end{array}$ & $\begin{array}{l}-0,3 \\
-0,8\end{array}$ & $\begin{array}{l}-0,3 \\
-0,2\end{array}$ & $\begin{array}{l}-9,1 \\
-8,1\end{array}$ \\
\hline $\begin{array}{c}>100 \\
\text { ENTR. } \\
\text { EMPLOI }\end{array}$ & $\begin{array}{r}-0,3 \\
2,9\end{array}$ & $\begin{array}{l}1,0 \\
1,8\end{array}$ & $\begin{array}{l}-1,3 \\
-0,8\end{array}$ & $\begin{array}{l}-1,1 \\
-0,4\end{array}$ & $\begin{array}{l}0,1 \\
0,5\end{array}$ & $\begin{array}{l}0,6 \\
2,1\end{array}$ & $\begin{array}{l}3,3 \\
4,1\end{array}$ & $\begin{array}{l}1,5 \\
2,4\end{array}$ & $\begin{array}{r}+3,9 \\
+13,3\end{array}$ \\
\hline $\begin{array}{l}\text { TOTAL } \\
\text { ENTR. } \\
\text { EMPLOI. }\end{array}$ & $\begin{array}{l}2,6 \\
2,2\end{array}$ & $\begin{array}{l}1,5 \\
1,2\end{array}$ & $\begin{array}{r}0,7 \\
-0,7\end{array}$ & $\begin{array}{l}0,9 \\
0\end{array}$ & $\begin{array}{l}1,6 \\
0,4\end{array}$ & $\begin{array}{l}1,9 \\
1,3\end{array}$ & $\begin{array}{l}1,9 \\
3,0\end{array}$ & $\begin{array}{l}1,9 \\
2,5\end{array}$ & $\begin{array}{l}+13,6 \\
+10,2\end{array}$ \\
\hline & 79 & 80 & 81 & 82 & 83 & 84 & 85 & 86 & $\Delta 78 / 86$ \\
\hline $\begin{array}{l}\text { TOTAL } \\
\text { SECTEURS } \\
1 \text { à } 49 \\
\text { ENTR. } \\
\text { EMPLOI }\end{array}$ & $\begin{array}{l}1,4 \\
0\end{array}$ & $\begin{array}{r}0,5 \\
-1,0\end{array}$ & $\begin{array}{l}-0,7 \\
-2,9\end{array}$ & $\begin{array}{l}-0,4 \\
-1,3\end{array}$ & $\begin{array}{r}0,7 \\
-0,7\end{array}$ & $\begin{array}{r}1,0 \\
-0,3\end{array}$ & $\begin{array}{l}1,6 \\
2,1\end{array}$ & $\begin{array}{l}1,6 \\
2,7\end{array}$ & $\begin{array}{r}+5,9 \\
-1,6\end{array}$ \\
\hline $\begin{array}{c}50 \text { à } 99 \\
\text { ENTR. } \\
\text { EMPLOI }\end{array}$ & $\overline{0}, 3$ & $\begin{array}{l}-3,0 \\
-2,8\end{array}$ & $\begin{array}{l}-5,3 \\
-5,1\end{array}$ & $\begin{array}{l}-4,8 \\
-5,3\end{array}$ & $\begin{array}{l}-4,9 \\
-4,7\end{array}$ & $\begin{array}{l}-1,8 \\
-0,7\end{array}$ & $\begin{array}{l}-1,7 \\
-2,2\end{array}$ & $\begin{array}{l}-1,3 \\
-0,5\end{array}$ & $\begin{array}{l}-20,8 \\
-19,3\end{array}$ \\
\hline $\begin{array}{c}>100 \\
\text { ENTR. } \\
\text { EMPLOI }\end{array}$ & $\begin{array}{l}-2,6 \\
-0,5\end{array}$ & $\begin{array}{l}-0,5 \\
-0,5\end{array}$ & $\begin{array}{l}-4,0 \\
-4,5\end{array}$ & $\begin{array}{l}-2,9 \\
-3,2\end{array}$ & $\begin{array}{l}-2,5 \\
-2,0\end{array}$ & $\begin{array}{l}-2,0 \\
-0,8\end{array}$ & $\begin{array}{r}+2,0 \\
0,3\end{array}$ & $\begin{array}{l}-1,9 \\
-0,6\end{array}$ & $\begin{array}{l}-13,5 \\
-11,2\end{array}$ \\
\hline $\begin{array}{l}\text { TOTAL } \\
\text { ENTR. } \\
\text { EMPLOI }\end{array}$ & $\begin{array}{l}-1,3 \\
-0,2\end{array}$ & $\begin{array}{r}0,4 \\
-0,8\end{array}$ & $\begin{array}{l}-0,8 \\
-4,0\end{array}$ & $\begin{array}{l}-0,6 \\
-2,7\end{array}$ & $\begin{array}{r}0,6 \\
-1,7\end{array}$ & $\begin{array}{r}0,9 \\
-0,6\end{array}$ & $\begin{array}{l}1,5 \\
0,8\end{array}$ & $\begin{array}{l}1,5 \\
0,6\end{array}$ & $\begin{array}{r}+5,0 \\
-8,5\end{array}$ \\
\hline
\end{tabular}




\section{Conclusions}

$\mathrm{Si}$, dans une perspective Shumpeterienne, la théorie économique semblait s'accorder à vanter les mérites et les avantages de la grande dimension par rapport à la petite, elle a, depuis, dû s'interroger sur les causes et les raisons du développement des petites et moyennes entreprises. Dans une conjoncture de mutation, la petite dimension montre qu'elle dispose de sérieux atouts et qu'elle constitue un élément essentiel d'évolution économique. A l'heure actuelle, de nouvelles perspectives lui sont incontestablement ouvertes, dont celle de participer activement à la troisième révolution industrielle.

La transformation des marchés, le développement de la sous-traitance, l'externalisation des économies d'échelle au niveau de l'organisation, le changement dans la nature des inventions et des innovations tendent à relativiser le rôle des grandes entreprises et à donner de nombreux avantages à la petite dimension.

L'analyse de la situation en Belgique a révélé non seulement le maintien de l'importance des petites entreprises mais aussi l'accroissement de cette importance relative dans une période de crise économique et de croissance zéro. Les P.M.E. ont incontestablement une meilleure résistance aux effets de la crise, qui repose partiellement sur leur plus grande souplesse d'adaptation face aux rigidités des grandes entreprises. Elles ont, par ailleurs, contribué ces toutes dernières années, à la création d'emplois, tant au niveau du secteur secondaire que tertiaire.

Les recherches effectuées dans diverses régions prouvent que les P.M.E. s'avèrent également particulièrement aptes à diffuser les progrès technologiques (Bollard, 1983, Oakey et Rothwell, 1984, Hagen, 1982, CEE, 1986, US National Science Foundation, 1978, Robson et Towsend, 1984, Wtterwulghe, 1986) et à contribuer ainsi à la création d'emplois. Le rôle qu'elles jouent, par ailleurs, dans le renouveau du tissu industriel est important. C'est grâce à une création nette de petites entreprises que bon nombre d'économies, dont la Belgique, parviennent à se développer.

Les P.M.E. sont, par leurs caractéristiques propres, un élément moteur de nos économies en mutation. Il convient, en conséquence, de créer un environnement économique propice à leur développement. De nombreux pays l'ont compris. La Commission Européenne a enfin défini un programme d'action pour les P.M.E. Celui-ci prévoit la création d'un environnement favorable à la petite dimension dans la perspective du grand marché intérieur Européen ainsi qu' une contribution positive aux besoins de flexibilité des P.M.E., qu'il s'agisse de problèmes liés à la création d'entreprises nouvelles ou à celui du développement deP.M.E. existantes. On ne peut que souhaiter une harmonisation des politiques industrielles européennes pour favoriser la petite dimension qui constitue le passage obligé du développement et de la créativité européenne. 


\section{BIBLIOGRAPHIE}

BAIN, J., International Differences and Industrial Structure, Yale University Press, 1966.

BIRCH, B., The Job Generation Process, Full Report M.I.T., Cambridge, Mas, 1979.

BOLLARD, A., Small Beginnings, London, Intermediate Technology Publications, 1983.

BOLTON, J.E., Small Firms, Report of the Committee of Enquiry on Small Firms, London, HMSO, reprinted 1978.

CURRAN, J. and ST ANWORTH, J., Bolton Ten Years on. A Research Inventory and Critical Review in Perspectives on a Decade of Small Business Research, Alderslot, Gower, 1984.

DELATTRE, M. et EYMARD DUVERNAY, F., Le Progrès des P.M.E. dans la crise : signe d'un relâchement du tissu industriel, Critiques de l' Economie Politique, $\mathrm{n}^{\circ} 26$ et 27, 1984.

DELATTRE, M., Le progrès des P.M.E. dans «La crise» in : La P.M.E. dans un monde en mutation, sous la direction de JULIEN, P.A., CHICHIN, J., JOYAL, A., Presses Universitaires du Québec, 1986.

DUPRIEZ, P., KUMPS, A.M. et WTTERWULGHE, R., Tableau de Bord Socio-Economique Bruxellois, Centre d'Etudes Regionales Bruxelloises, Bruxelles, 1987.

FOTHERGILL, S. and GUDGIN, G., The Job Generation Process in Britain, London, Centre for Environmental Studies, 1979.

GALBRAITH, J., The New Industrial State, Boston, Houghton Nifflin, 1967.

GREFFE, X., Les P.M.E. créent-elles des emplois ?, Paris, Economica, 1984.

HAGEN, E., Technological Disemployment and Economic Growth, Journal of Development 1982, n 10, pp. 171-186.

JULIEN, P.A. et MOREL, B., La Belle Entreprise, Québec, Boréal, 1986.

MARX, K., Le Capital, Paris, la Pléiade, 1968.

OAKEY, R.P., and ROTHWELL, R., High Technology Small Firms and Regional Growth, Centre for Regional and Urban Studies, University of Newcastle, March 1984.

PENROSE, E., The Theory of the Growth of the Firm, Oxford, Basil Blackwell, 1959.

ROBSON, M. and TOWSEND, J., Trends and Characteristics of Significant Innovations and their Innovators in the United Kingdom since 1945, S.P.R.U., University of Sussex, August 1984.

SAMUELS, S.J. and SMYTH, D.J., Profits, Variability of Profit and Firm Size, Economica, May 1968.

SCHERER, F.M., Industrial Market Structure and Economic Performance, Chicago, Rand Mc Nelly, 1970.

SHUMPETER, J., Capitalism, Socialism and Democracy, New York, Harper, 1947.

STOREY, D., Job Generation and Small Firm Policy in Britain, Centre for Environmental Studies, London, Research Series 11, 1980.

WTTERWULGHE, R., The New Technologies and the Small and Medium Sized Enterprises in S.M.E.: Keystone for a Free and Prosperous Europe, European Research and Documentation Centre, Carolus Magnus, Tilburg University Press, 1986.

Commission des Communautés Européennes, l'Amélioration de la compétitivité et des structures industrielles de la Communauté. Communication au Conseil (86) 40 final, février 1986.

U.S. National Science Foundation, Research and Development in Industry, 1978.

Un programme d'action pour les P.M.E., Europe, documents n 1418/1419, 18-9-86. 